\title{
Thyroid disease and cancer in kidney transplantation: a single-center analysis
}

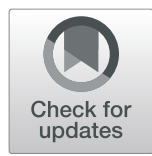

\author{
Massimiliano Veroux ${ }^{1 *} \mathbb{D}$, Giuseppe Giuffrida ${ }^{2}$, Salvatore Lo Bianco ${ }^{3}$, Matteo Angelo Cannizzaro ${ }^{4}$, Daniela Corona ${ }^{5}$, \\ Alessia Giaquinta ${ }^{6}$, Chiara Palermo ${ }^{6}$, Fausto Carbone ${ }^{6}$, Anna Carbonaro $^{6}$, Maria Teresa Cannizzaro7, \\ Rossella Gioco ${ }^{6}$ and Pierfrancesco Veroux ${ }^{6}$
}

\begin{abstract}
Background: Thyroid diseases are frequent in patients with end-stage renal disease, but data on renal transplant recipients are conflicting. This study evaluated the incidence of thyroid disease and cancer in a population of kidney transplant recipients performed in a single center.

Methods: Seven hundred sixty patients receiving a kidney transplantation between January 2000 and October 2017 were followed with thyroid ultrasonography to determine nodules together with thyroid hormone levels. Ultrasound-guided fine-needle aspiration citology (FNAc) was performed to the nodules $>10 \mathrm{~mm}$.

Results: Two hundred four patients (26.8\%) patients demonstrated functional or morphologic changes in the thyroid gland compared with pre-transplant period. Among the 204 patients with newly diagnosed thyroid disease, 165 patients had single or multiple nodular lesions less than $1 \mathrm{~cm}$ in diameter, and were followed yearly. Nodule size progression was observed in 23 patients (13.9\%), and they underwent a FNAc. A total of sixty-two patients (30.3\%) underwent FNAc. The biopsy samples were cytologically interpreted as benign in 20 patients (32.2\%), suspicious in 40 patients (64.5\%), or at high risk of cancer in 2 patients (3.2\%). Forty-two patients underwent total thyroidectomy. At histological examination, 18 patients had a thyroid cancer (papillary cancer in 17 patients, follicular cancer in one). Thyroid cancer was more frequent in male patients with a mean time from transplant to diagnosis of 5.6 years. At a mean follow-up was $8 \pm 1.2$ years, all patients are alive with a normal functioning graft.

Conclusions: Thyroid diseases are common in transplant recipients. Thyroid disease may evolve after transplantation, probably as a consequence of immunosuppression. A complete evaluation of thyroid disease is mandatory in kidney transplant recipients because early diagnosis and appropriate treatment of thyroid disease and cancer may significantly decrease the morbidity and mortality in these patients.
\end{abstract}

Keywords: Thyroid, Goiter, Kidney transplantation, Cancer, Thyroid nodule, Papillary, Follicular, Fine needle aspiration citology

\section{Background}

Kidney transplantation is the preferred replacement therapy for patients with end-stage renal disease (ESRD). However, long-term immunosuppression may be responsible of a variety of complications, including the development of thyroid disease and cancer [1, 2]. Many studies

\footnotetext{
* Correspondence: veroux@unict.it

${ }^{1}$ Vascular Surgery and Organ Transplant Unit, Unit of Endocrine Surgery, Department of Medical and Surgical Sciences and Advanced Technologies, University Hospital of Catania, Via Santa Sofia, 8495123 Catania, Italy Full list of author information is available at the end of the article
}

have shown that, in kidney transplant recipients, the most common thyroid imbalance is a low T3 syndrome with FT3 levels generally within the normal limits [2-5], and this may have an impact on graft survival $[5,6]$. Moreover, thyroid disease in kidney transplantation has been identified as a risk factor for cardiovascular disease (CVD) and a predictor of mortality [7].

Thyroid diseases may worsen after transplantation, mainly due to a consequence of the immunosuppression and this may probably explain the increased risk of thyroid cancer after transplantation [2, 8-12]. Interestingly,

(c) The Author(s). 2019 Open Access This article is distributed under the terms of the Creative Commons Attribution 4.0 International License (http://creativecommons.org/licenses/by/4.0/), which permits unrestricted use, distribution, and 
the risk of cancer in patients with ESRD is higher during dialysis period than during functioning kidney transplant intervals [13]. Taken together, these data suggest that a periodical evaluation of thyroid function should be recommended in kidney transplant recipients, but there are few studies investigating the rate of thyroid disease in kidney transplant recipients.

This study evaluated the incidence of thyroid disease and cancer in a population of kidney transplant recipients performed in a single center.

\section{Methods}

This retrospective study included 760 patients who received kidney transplantation between January 2000 and October 2017. All ESRD patients, to be included in the waiting list, underwent ultrasonography of the neck and complete assessment of thyroid function according to serum levels of FT3, FT4, and TSH, as previously described [2]. In brief, all patients with a benign thyroid disease (nodular goiter or thyroiditis) were considered eligible for kidney transplantation after treatment of the disease, while patients with a diagnosis of thyroid cancer were considered eligible for kidney transplantation after at least 2 years of negative follow-up [2].

Kidney transplant recipients received a standard three-drug immunosuppressive therapy, with or without induction therapy with anti-interleukin-2 receptor antibodies (Simulect, Novartis, Basel, Switzerland) or with antithymocyte globulin (ATG-Fresenius, Fresenius, Bad Homburg, Germany), basing on both donor and recipient characteristics, as previously described [14].

After transplantation, in all transplant recipients Free T3 (normal range 1.8-4.2 pg/mL) and free T4 (normal range $0.8-1.9 \mathrm{ng} / \mathrm{dL}$ ) levels were measured on a yearly basis and an ultrasonography of the neck was performed every 2 years. Anti-thyroid peroxidase antibody, anti-thyroglobulin antibody (Anti Tg), and serum TSH levels were also measured on a yearly basis. All patients with an isoechogenic solid thyroid nodule greater than $1 \mathrm{~cm}$ in diameter underwent fine-needle aspiration cytology (FNAc). Each cytological specimen was smeared on a slide immediately after aspiration. Thyroid nodules were classified according to the Italian cytological classification [15].

All patients with high levels of FT3 and FT4, low levels of TSH, and clinical signs or symptoms of hyperfunction of the thyroid gland (eg, tachycardia, flushing, or anxiety) underwent scintigraphy with iodine 131.

Surgical treatment was performed in kidney transplant recipients with a diagnosis of thyroid cancer or an uncertain diagnosis at FNAc, follicular adenoma, Plummer adenoma, or multinodular goiter and compressive symptoms on adjacent organs. Patients with benign disease, cystic lesions, or thyroid nodules smaller than $1 \mathrm{~cm}$ were followed up on yearly basis to evaluate thyroid function and perform ultrasonography [2].

\section{Statistical analysis}

Results and patients characteristics are reported as mean \pm SD. Comparison of means and percentages was estimated by the unpaired two Student's t-test or Mann-Whitney $\mathrm{U}$ test, as appropriate. $p$ value $<0.05$ was considered as statistically significant. All calculations were performed using SPSS, version 12.0.

\section{Results}

Two hundred four patients (26.8\%) patients demonstrated functional or morphologic changes in the thyroid gland compared with pre-transplant period. The demographical and clinical characteristics of patients are reported in Table 1. Clinical characteristics were similar among recipients developing a thyroid nodule compared to recipients who did not. Patients with thyroid nodule were more frequently younger with a longer time on dialysis and on waiting list for kidney transplantation, suggesting the role of time on dialysis on the development of thyroid disease. Type of immunosuppression did not have a role in the development of thyroid disease.

Among the 204 patients with newly diagnosed thyroid disease, 165 patients had single or multiple nodular lesions less than $1 \mathrm{~cm}$ in diameter, and were followed

Table 1 Clinical characteristics of the study cohort

\begin{tabular}{|c|c|c|c|}
\hline & $\begin{array}{l}\text { With nodule } \\
(n=204)\end{array}$ & $\begin{array}{l}\text { Without nodule } \\
(n=556)\end{array}$ & P \\
\hline Male (\%) & $48 \%$ & $47 \%$ & Ns \\
\hline Living/Deceased & $40 / 164$ & $60 / 494$ & Ns \\
\hline Age (years) & $48 \pm 12.3$ & $54 \pm 10.5$ & $<0.04$ \\
\hline \multicolumn{4}{|l|}{ Cause of ESRD $(n, \%)$} \\
\hline Glomerulonephritis & $95(46.6)$ & $265(47.6)$ & Ns \\
\hline $\begin{array}{l}\text { Polycystic Kidney } \\
\text { Disease }\end{array}$ & $40(19.7)$ & $87(15.7)$ & Ns \\
\hline Diabetes & $30(14.7)$ & $60(10.8)$ & Ns \\
\hline Other causes & $39(19.1)$ & $144(25.9)$ & Ns \\
\hline Waiting list (months) & $21 \pm 10.4$ & $18.2 \pm 16.4$ & Ns \\
\hline Pretransplant dialysis & $32 \pm 16.3$ & $29 \pm 22.3$ & $<0.05$ \\
\hline Donor age & $55.6 \pm 15.1$ & $54.2 \pm 18.1$ & Ns \\
\hline \multicolumn{4}{|l|}{ Immunosuppression (n, \%) } \\
\hline Induction & $60(29.4)$ & $160(28.2)$ & Ns \\
\hline TAC+MPA+Ster & $170(83.3)$ & $470(83)$ & Ns \\
\hline CYA+MPA+Ster & $20(9.8)$ & $60(10.6)$ & Ns \\
\hline Eve+MPA+Ster & $24(11.7)$ & $36(6.3)$ & Ns \\
\hline $\mathrm{BMI}\left(\mathrm{KG} / \mathrm{m}^{2}\right)$ & $25 \pm 4.7$ & $26.1 \pm 3.9$ & Ns \\
\hline Serum Creatinine $(\mathrm{mg} / \mathrm{dL})$ & $1.53 \pm 0.7$ & $1.49 \pm 0.6$ & Ns \\
\hline
\end{tabular}

ESRD end-stage renal disease, TAC tacrolimus, MPA mycophenolic acid, Ster steroids, $C y A$ cyclosporine, $E V E$, everolimus, $B M I$ body mass index NS, not significant 
yearly. Nodule size progression was observed in 23 patients (13.9\%), and they underwent a FNAc.

A total of sixty-two patients (30.3\%) underwent FNAc: Of the patients who had nodules, 58 patients had normal thyroid function, while clinical hyperthyroidism was diagnosed in 4 patients. The biopsy samples were cytologically interpreted as benign (TIR 2) in 20 (32.2\%), suspicious (TIR 3A or B) in 40 patients $(64.5 \%)$, or at high risk of cancer (TIR 4) in 2 patients (3.2\%).

Two patients with clinical signs of hyperthyroidism at 131I scintigraphy had a hyperfunctional goiter and underwent left lobectomy.

Forty-two patients underwent total thyroidectomy. At histological examination, 18 patients had a thyroid cancer (papillary cancer in 17 patients, follicular cancer in one). Patients with thyroid cancer were more frequently male (15/18), with a median age of $42 \pm 15.4$ years. Mean time from transplant to diagnosis was 5.6 y (range 3-12, years). Multifocal cancer was observed in three patients, while microcarcinoma $(<1 \mathrm{~cm})$ was observed in three patients. Four patients presented with lymph node metastasis at the time of diagnosis.

Adjuvant radioiodide treatment was applied in all patients with persistent high level of thyroglobulin after thyroidectomy. In patients with diagnosis of cancer, immunosuppressive therapy was slightly reduced without the need for a switching to another drug.

Three patients, with lymph node metastasis at time of diagnosis, had a local recurrence at 6,10 and 11 months after thyroidectomy and underwent lymph node dissection. There were no signs of local recurrence or distant metastasis in the remaining 15 patients. At a mean follow-up was $8 \pm 1.2$ years, all patients are alive with a normal functioning graft.

\section{Discussion}

Thyroid disease is frequent in patients with end-stage renal disease, but data on renal transplant recipients are conflicting. The uremia and the long-term immunosuppression may have a role in the increased incidence of thyroid carcinoma in renal transplant patients compared to general population [16].

However, very few studies investigated the incidence of thyroid disease in kidney transplant population. In a study by Łebkowska $U$ et al., [17], the incidence of thyroid disease was evaluated by ultrasound-guided FNAb in 44 kidney transplant recipients. They found that all patients presented with a various degree of goiter, with a thyroid nodule in $43 \%$ of patients and a thyroid cancer in $11.4 \%$ of patients. Moreover, they found a significant association between cyclosporine level and the occurrence of goiter, confirming that higher level of immunosuppression may increase the risk of developing a thyroid cancer [17]. More recently, Gungor et al [16], reported a higher incidence of thyroid nodule $(40 \%)$ and goiter $(91.8 \%)$ in 122 kidney transplant recipients compared with the general population.

This study evaluated the incidence of thyroid disease among a population of 760 kidney transplantations performed in a single center. A newly diagnosed thyroid disease was reported in 240 patients (16.8\%). Most of patients had thyroid nodule $<1 \mathrm{~cm}$ in diameter $(80.8 \%)$, but 18 patients developed a thyroid cancer. Younger patients, with long-term dialysis and waiting list are at higher risk for developing a thyroid cancer, but there was no significant association with the type of immunosuppression adopted, suggesting that the cumulative dose of immunosuppression rather than the type of immunosuppression may have a significant role in the increased risk of thyroid cancer in kidney transplant patients.

Organ transplant recipients have higher risk of developing a post-transplant cancer. However, this risk is particularly high for virus-linked neoplasm, in which some oncogenic viruses such as human herpesvirus-8 or Epstein-Barr virus are activated by immunosuppression causing the post-transplant lymphoproliferative disorders. In contrast, cancers that are more frequently observed in the general population (colon, breast, lung, etc) are not related to oncovirus, and their incidence is not significantly increased after renal transplantation [2, 8-13].

There are few data in literature on the incidence of thyroid cancer in kidney transplant recipients. A study from Australia and New Zealand [18] evaluated the incidence of thyroid cancer among a cohort of 10,689 renal transplant recipients, and found that 23 patients $(0.22 \%)$ were diagnosed with a post-transplant thyroid cancer. The median age in the renal transplant group with thyroid cancer was 48.2 years. Interestingly, thyroid cancer was more common in male sex, as confirmed in our study, and $43 \%$ of patients presented lymphatic metastases at the time of the primary diagnosis. More recently, Karamchandani et al [8] showed that the risk of thyroid cancer is significantly higher following kidney transplantation (SIR 6.94), and most cancers are of papillary type and appear about 6 years following transplant. More recent studies reported a SIR of 1.9 [10], while Kitahara et al [11] reported an incidence rate ratio of thyroid cancer among kidney transplant recipients of 1.26 , being more frequent when the cause of ESRD was hypertensive nephrosclerosis and in patients with longer prior dialysis before transplantation. Overall, thyroid cancer was associated with a modest increase of death (hazard ratio 1.33).

The incidence of thyroid cancer in our series was $2.3 \%$. All patients but one had a papillary cancer. Although rarely observed, follicular neoplasm may occur even in kidney transplant recipients, and pre-operative 
diagnosis is often difficult due to the limited sensitivity of FNAc, mandating a surgical treatment in most patients [19]. A lymphatic metastasis was observed in four patients, and cancer occurred more frequently in men at a mean time of 5.6 years after transplantation. In general population, lymph nodal involvement is very common in differentiated thyroid cancer, with micrometastases observed in up to $80 \%$ of papillary thyroid cancers [20], but this higher incidence was not observed in our series, probably due to early diagnosis. Thyroid cancer recurred in three patients, but patient and graft survival were not significantly affected and all patients are alive at last follow-up. Again, the chronic immunosuppression may be likely related to the risk of thyroid cancer, as demonstrated by the occurrence of cancer many years after the transplantation. However, recent studies have demonstrated that thyroid cancer can occur even early in the post-transplantation follow-up and its histologic behavior is often more aggressive than in the general population [2].

Although the more aggressive behavior of thyroid cancer in kidney transplant recipients, there was no cancer-related mortality in our experience. We could suggest, therefore, a yearly-based neck sonography in all kidney transplant recipients together with the evaluation of thyroid functionality. In all patients with a nodule increasing in size or suggestive for thyroid cancer at citology, a total thyroidectomy should be performed.

The main limit of this study is that, although performed in a large cohort of patients, many patients could be lost at follow up, due to graft loss or patient death, so that the incidence of thyroid disease and cancer could be underestimated.

\section{Conclusions}

Thyroid diseases are common in transplant recipients. Thyroid disease may evolve after transplantation, probably as a consequence of immunosuppression. A complete evaluation of thyroid disease in kidney transplant recipients is mandatory because early diagnosis and appropriate treatment of thyroid disease and cancer may significantly decrease the morbidity and mortality in these patients.

\section{Abbreviations}

ATG: Antithymocyte globulin; CVD: Cardiovascular disease; ESRD: End-stage renal disease; FNAb: Fine-needle aspiration biopsy; FNAc: Fine-needle aspiration cytology; TSH: Thyroid stimulant hormone

\section{Funding}

Publication of this article was funded by the Specialty School in General Surgery of the University of Catania.

\section{Availability of data and materials}

All data generated or analysed during this study are available from the corresponding author on reasonable request.

\section{About this supplement}

This article has been published as part of BMC Surgery Volume 18 Supplement 1 , 2018: Updates and New Technology in Endocrine Surgery. The full contents of the supplement are available online at https://bmcsurg.biomedcentral.com/articles/ supplements/volume-18-supplement-1.

\section{Authors' contributions}

MV, MAC, AG, GG, DC, PV conceived the study, wrote the draft of the article $C P, F C, A C, M T C, S L B, R G$ collected the data and performed the statistical analysis, MV, MAC wrote the final version of the manuscript. All the authors read and gave the final approval to the manuscript.

\section{Ethics approval and consent to participate}

This study is an observational study and the ethical committee of the University Hospital of Catania ruled that no formal ethical approval was required in this particular cases. However, all patients signed an informed consent form for diagnostic and surgical procedures.

\section{Consent for publication}

Not applicable.

\section{Competing interests}

The authors declare that they have no competing interests.

\section{Publisher's Note}

Springer Nature remains neutral with regard to jurisdictional claims in published maps and institutional affiliations.

\section{Author details}

'Vascular Surgery and Organ Transplant Unit, Unit of Endocrine Surgery, Department of Medical and Surgical Sciences and Advanced Technologies, University Hospital of Catania, Via Santa Sofia, 8495123 Catania, Italy. ${ }^{2}$ Manchester Royal Infirmary Organ Transplant Unit, Manchester, UK. ${ }^{3}$ Unit of Endocrine Surgery, University Hospital of Catania, Catania, Italy. ${ }^{4}$ Unit of Endocrine Surgery, Department of Medical and Surgical Sciences and Advanced Technologies, University Hospital of Catania, Catania, Italy. ${ }^{5}$ Vascular Surgery and Organ Transplant Unit, Department of Medical and Surgical Sciences, University Hospital of Catania, Catania, Italy. ${ }^{6}$ Vascular Surgery and Organ Transplant Unit, University Hospital of Catania, Catania, Italy. 'Radiology Unit, University Hospital of Catania, Catania, Italy.

Received: 17 August 2018 Accepted: 11 September 2018 Published: 24 April 2019

\section{References}

1. Veroux M, Corona D, Veroux P. Kidney transplantation: future challenges. Minerva Chir. 2009:64:75-100

2. Veroux M, Giuffrida G, Gagliano M, Giaquinta A, Tallarita T, Sorbello M, Corona D, Zerbo D, Vizcarra D, Scriffignano V, Cannizzaro MA, Veroux P. Evaluation of thyroid disease in kidney transplantation candidates: management and follow-up. Transplant Proc. 2009:41:1142-4

3. Rotondi M, Netti GS, Rosati A, Mazzinghi B, Magri F, Ronconi E, Becherucci F, Pradella F, Salvadori M, Serio M, Romagnani P, Chiovato L. Pretransplant serum FT3 levels in kidney graft recipients are useful for identifying patients with higher risk for graft failure. Clin Endocrinol. 2008;68:220-5.

4. Junik R, Wlodarczyk Z, Masztalerz M, Odrowaz-Sypniewska G, Jendryczka E, Manitius J. Function, structure, and volume of thyroid gland following allogenic kidney transplantation. Transplant Proc. 2003:6:2224-6.

5. Iglesias P, Bajo MA, Selgas R, Díez JJ. Thyroid dysfunction and kidney disease: an update. Rev Endocr Metab Disord. 2017;18:131-44.

6. Song SH, Kwak IS, Lee DW, Kang YH, Seong EY, Park JS. The prevalence of low triiodothyronine according to the stage of chronic kidney disease in subjects with a normal thyroidstimulating hormone. Nephrol Dial Transplant. 2009:5:1534-8.

7. Chonchol M, Lippi G, Salvagno G, Zoppini G, Muggeo M, Targher G. Prevalence of subclinical hypothyroidism in patients with chronic kidney disease. Clin J Am Soc Nephrol. 2008:5:1296-300.

8. Karamchandani D, Arias-Amaya R, Donaldson N, Gilbert J, Schulte KM. Thyroid cancer and renal transplantation: a meta-analysis. Endocr Relat Cancer. 2010;17:159-67. 
9. Veroux M, Puliatti C, Fiamingo P, Cappello D, Macarone M, Puliatti D, Vizcarra D, Gagliano M, Veroux P. Early de novo malignancies after kidney transplantation. Transplant Proc. 2004;36:718-20.

10. Piselli P, Serraino D, Segoloni GP, Piselli P, Serraino D, Segoloni GP, Sandrini S, Piredda GB, Scolari MP, Rigotti P, Busnach G, Messa P, Donati D, Schena FP, Maresca MC, Tisone G, Veroux M, Sparacino V, Pisani F, Citterio F. Immunosuppression and Cancer study group. Risk of de novo cancers after transplantation: results from a cohort of 7217 kidney transplant recipients, Italy 1997-2009. Eur J Cancer. 2013:49:336-44.

11. Kitahara CM, Yanik EL, Ladenson PW, Hernandez BY, Lynch CF, Pawlish KS, Engels EA. Risk of thyroid Cancer among solid organ transplant recipients. Am J Transplant. 2017;17:2911-21.

12. Hortlund M, Arroyo Mühr LS, Storm H, Engholm G, Dillner J, Bzhalava D. Cancer risks after solid organ transplantation and after long-term dialysis. Int J Cancer. 2017:140:1091-101.

13. Yanik EL, Clarke CA, Snyder JJ, Pfeiffer RM, Engels EA. Variation in cancer incidence among patients with ESRD during kidney function and nonfunction intervals. J Am Soc Nephrol. 2016;5:1495-504.

14. Veroux M, Zerbo D, Basile G, Gozzo C, Sinagra N, Giaquinta A, Sanfiorenzo A, Veroux P. Simultaneous native nephrectomy and kidney transplantation in patients with autosomal dominant polycystic kidney disease. PLoS One. 2016:11(6):e0155481.

15. Tartaglia F, Giuliani A, Tromba L, Carbotta S, Karpathiotakis M, Tortorelli G, Pelle F, Merola R, Donello C, Carbotta G, De Anna L, Conzo G, Sorrenti S, Ulisse S. Fine needle aspiration cytology of 650 thyroid nodules operated for multinodular goiter: a cyto-histological correlation based on the new Italian cytological classification (siapec 2014). J Biol Regul Homeost Agents. 2016:30:1187-93.

16. Gungor O, Celik A, Kebapcilar L, Karaoglu O, Ersan S, Atilla K, Canda T, Bayraktar $F$, Yesil S. Incidence of thyroid dysfunction and thyroid cancer in renal transplant recipients: a single center experience. Ren Fail. 2010; 32:167-71.

17. Łebkowska U, Małyszko JS, Małyszko J, Dziecioł J, Walecki J, Myśliwiec M. Thyroid function and incidentalomas in kidney transplant recipients. Med Sci Monit. 2003;9:MT8-MT11.

18. Pond F, Serpell JW, Webster A. Thyroid cancer in the renal transplant population: epidemiological study. ANZ J Surg. 2005;75:106-9.

19. Conzo G, Avenia N, Ansaldo GL, Calò P, De Palma M, Dobrinja C, Docimo G, Gambardella C, Grasso M, Lombardi CP, Pelizzo MR, Pezzolla A, Pezzullo L, Piccoli M, Rosato L, Siciliano G, Spiezia S, Tartaglia E, Tartaglia F, Testini M, Troncone G, Signoriello G. Surgical treatment of thyroid follicular neoplasms: results of a retrospective analysis of a large clinical series. Endocrine. 2017; 55:530-8.

20. Gambardella C, Tartaglia E, Nunziata A, Izzo G, Siciliano G, Cavallo F, Mauriello C, Napolitano S, Thomas G, Testa D, Rossetti G, Sanguinetti A, Avenia N, Conzo G. Clinical significance of prophylactic central compartment neck dissection in the treatment of clinically node-negative papillary thyroid cancer patients. World J Surg Oncol. 2016;14:247.

Ready to submit your research? Choose BMC and benefit from:

- fast, convenient online submission

- thorough peer review by experienced researchers in your field

- rapid publication on acceptance

- support for research data, including large and complex data types

- gold Open Access which fosters wider collaboration and increased citations

- maximum visibility for your research: over $100 \mathrm{M}$ website views per year

At BMC, research is always in progress.

Learn more biomedcentral.com/submissions 